\title{
Influencia del calcio en la retención de humedad por arcilla
}

\author{
Influence of calcium in moisture \\ retention clay
}

\author{
Natalia Castrillo ${ }^{1,2}$, Adela Mercado ${ }^{2,3}$ \\ Cristina Volzone ${ }^{4}$
}

\author{
${ }^{1}$ INIQUI-CONICET, Universidad Nacional de Salta CP: 4400, Salta, Salta, Argentina. \\ e-mail: ncastrillo@unsa.edu.ar \\ ${ }^{2}$ CIUNSA, Universidad Nacional de Salta, CP: 4400, Salta, Salta, Argentina. \\ ${ }^{3}$ FAC. DE CIENCIAS EXACTAS,Universidad Nacional de Salta, CP: 4400, Salta, Salta, Argentina. \\ e-mail: adelaisabelmercado@gmail.com \\ ${ }^{4}$ CETMIC (CIC PBA, CCT CONICET La Plata), CP: 1897, M.B. Gonnet, Buenos Aires, Buenos Aires, Argentina. \\ e-mail: cvolzone@cetmic.unlp.edu.ar
}

\section{RESUMEN}

La bentonita como materia prima constituye una alternativa económica para la producción de desecantes. La literatura indica que es un buen adsorbente de agua en relación a los desecantes comúnmente usados en la industria (ślica gel, tamiz molecular). En este sentido, constituye una alternativa válida y una opción económica ya que la arcilla es un compuesto natural, lo cual hace que sea amigable con el medio ambiente. De este modo, se consigue proteger productos de la humedad con la ventaja adicional de que su capacidad desecante se mantiene prácticamente constante luego de sucesivos ciclos de adsorción - regeneración, utilizándose baja temperatura $\left(100^{\circ} \mathrm{C}\right)$ para este último proceso. Teniendo en cuenta el interés en aumentar la explotación de bentonita en Argentina, en estudios previos se evaluó su capacidad de retención, utilizando entre otras, una bentonita de la Patagonia. La adsorción de vapor de agua es afectada por la concentración del mineral de arcilla esmectita, la humedad relativa, el secado de las muestras y el tipo de catión interlaminar de la esmectita. En el presente trabajo se determinó que existe un incremento de adsorción en relación al material de partida luego del agregado de concentraciones crecientes de Ca por la vía intercambio iónico. En estas muestras la adsorción se lleva a cabo principalmente por el ión hidratado presente en el espacio interlaminar. Asimismo, los materiales desecantes bentonita-Ca mostraron diferencias estructurales y texturales en relación a la bentonita natural.

Palabras clave: Arcilla,Calcio, Retención, Humedad.

\begin{abstract}
Bentonite as a raw material is an economical alternative to produce desiccants. The literature indicates that it is a good water adsorbent in relation to the desiccants commonly used in industry (silica gel, molecular sieve). In this sense, it is a valid alternative and an economic option since the clay is a natural compound, which makes it environmentally friendly. In this way, it is possible to protect products from moisture with the additional advantage that its desiccant capacity remains practically constant after successive cycles of adsorption - regeneration, using low temperatures $\left(100^{\circ} \mathrm{C}\right)$ for the latter process. Considering the interest of increasing the exploitation of bentonite in Argentina, previous studies evaluated its retention capacity, using, among others, a Patagonian bentonite. The adsorption of water vapor is affected by the concentration of the smectite clay mineral, the relative humidity, the drying of the samples, and the type of interlaminar cation of the smectite. In the present work it is determined that there is an increase of adsorption in relation to the starting material after the addition of increasing concentrations of $\mathrm{Ca}$ by ion exchange. In these samples the adsorption is carried out mainly by the hydrated ion present in the interlaminar space. Also, the desiccant materials bentonite-Ca showed structural and textural differences in relation to natural bentonite.
\end{abstract}

Keywords: Clay, Calcium, Retention, Moisture. 


\section{INTRODUCCIÓN}

La bentonita es una roca compuesta predominantemente por un mineral arcilloso del grupo de las esmectitas, la montmorillonita, asociada a impurezas tales como yeso, cuarzo y feldespatos [1]. Es un material con una mineralogía definida y propiedades fisicoquímicas determinadas [2], susceptibles a ser mejoradas por medio de modificaciones. La característica estructural más sobresaliente de las esmectitas es que diferentes moléculas pueden penetrar entre las láminas causando una expansión de la red. En particular, la adsorción de agua en la interlámina está gobernada por la naturaleza y carga del catión interlaminar, así como por el valor y localización de la carga laminar [3-6]. A una dada humedad relativa (HR), los cationes interlaminares se hidratan formando complejos de coordinación con valores d001 definidos. Al aumentar la HR los espacios sin ocupar entre los cationes hidratados son ocupados con moléculas de agua, ligándose entre sí por puentes de hidrógeno. Así, los cationes de cambio contribuyen fuertemente a la energía de hidratación interlaminar [7].

Los desecantes son agentes que exhiben una fuerte afinidad con la humedad que adsorben de la atmósfera. La deshumidificación contribuye a evitar problemas tales como corrosión, condensación, olores, moho, etc. Entre las industrias que utilizan desecantes se encuentran las de empaquetamiento de componentes electrónicos, farmacéuticos e industriales [8,9]. Los más usados por las mismas son gel de silicio, tamices moleculares y carbón activado. Durante los últimos años existió un creciente interés en utilizar materiales de bajo costo y de fácil acceso para el uso desecante, tales como sepiolita [10], caolinita [11], zeolita [12] y bentonita $[4,6,13]$, manifestando todos ellos eficacia dentro de un rango de temperatura y HR normal. Materiales bentoníticos modificados con Ca también son baratos y efectivos en el uso como desecante [11, 14, 15]. En particular, los materiales homoionizados con calcio requieren de protocolos de preparación muy sencillos, demandando bajos contenidos de $\mathrm{Ca}$ incorporado en la bentonita hasta compensar la capacidad de intercambio catiónico (CIC) del material natural. Teniendo en cuenta además que son los más eficientes en el proceso de adsorción de humedad [5].

En el presente trabajo se estudió una bentonita natural policatiónica de Rio Negro, identificada como BR, con el fin de estudiar sus propiedades y características antes y después de ser modificada mediante el proceso de intercambio iónico con $\mathrm{Ca}^{2+}$ y su aplicación en procesos de adsorción de agua en ambientes cerrados a $25^{\circ} \mathrm{C}$ y HR constante.

\section{MATERIALES Y MÉTODOS}

Antes de iniciar el desarrollo experimental se aseguró en la bentonita natural, BR, un tamaño de partícula pasante Malla $100(144 \mu \mathrm{m})$.

La capacidad de intercambio catiónico (CIC), propiedad fundamental que poseen las esmectitas, hace referencia a la posibilidad de intercambiar los cationes adheridos en la superficie exterior de sus cristales, en los espacios interlaminares o en otros espacios por cationes presentes en una solución acuosa en contacto con el mineral y es equivalente al total de cargas negativas de la estructura. La CIC de BR es de 100,7 meq/100 g, determinada en nuestro laboratorio.

\subsection{Caracterización}

La caracterización mineralógica y estructural fue realizada por difracción de rayos X (DRX) y espectroscopia infrarroja con transformada de Fourier (FTIR). La caracterización textural fue realizada mediante adsorcióndesorción de $\mathrm{N}_{2}$ y la composición química por fluorescencia de rayos X (FRX).

Los espectros DRX de las arcillas fueron obtenidos utilizando un equipo Philips PW 3710, 3kW, con radiación $\mathrm{Cu}-\mathrm{K}_{\alpha}, 45 \mathrm{kV}, 20 \mathrm{~mA}$, y rango de barrido ${ }^{\circ} 2 \theta$. Para la identificación mineralógica se utilizó la base de datos Powder Diffraction File.

Los estudios de FTIR se realizaron en un Spectrum GX Perkin Elmer. Los espectros de absorción se obtuvieron en la zona de $4000-400 \mathrm{~cm}^{-1}$. Las muestras, previamente secadas a $70^{\circ} \mathrm{C}$ durante $16 \mathrm{~h}$, se prepararon en pastillas soportadas en $\mathrm{KBr}$, compactadas a una presión de $2,5 \times 10^{16} \mathrm{~Pa}$.

Los estudios DTA-TG se realizaron en forma simultánea en un equipo Netsch STA 409. La velocidad de calentamiento fue de $10^{\circ} \mathrm{C} / \mathrm{min}$ en atmósfera de aire hasta $1200^{\circ} \mathrm{C}$.

Las isotermas de adsorción de $\mathrm{N}_{2}$ a $77 \mathrm{~K}$ se obtuvieron en un analizador Micromeritics ASAP 2020. Antes de la adsorción, cada muestra se desgasificó a $150^{\circ} \mathrm{C}$ en vacío durante $10 \mathrm{~h}$. La superficie específica $\left(\mathrm{S}_{\mathrm{BET}}\right)$ se estimó mediante el método de Brunauer, Emmet y Teller (BET), usando datos de adsorción en los rangos de presiones relativas $\left(\mathrm{p} / \mathrm{p}_{0}\right)$ donde se cumplen las condiciones y consideraciones del método [16].

La composición química fue determinada por fluorescencia de rayos X. El equipo utilizado fue un es- 
pectrómetro dispersivo en longitudes de onda, Tubo de Rh, de $3 \mathrm{~kW}$. Se trabajó a $50 \mathrm{kV}$ y $40 \mathrm{~mA}$. Las muestras se prepararon por fusión con tetraborato de $\mathrm{Li}$ en un crisol de $\mathrm{Pt} / \mathrm{Au}$ a $950^{\circ} \mathrm{C}$ a una relación muestra/ fundente de 1:10. Las curvas de calibrado se construyeron con patrones de referencia certificados. También se determinó la pérdida por calcinación (PPC) a $1000^{\circ} \mathrm{C}$.

\subsection{Modificación por Agregado de Calcio}

La bentonita policatiónica fue modificada por el agregado de la sal higroscópica $\mathrm{CaCl}_{2} \cdot 2 \mathrm{H}_{2} \mathrm{O}$, utilizando la vía húmeda de intercambio iónico. Los materiales compuestos se refirieron con el nombre de la bentonita, seguido del porcentaje en peso de Ca agregado. Así, por ejemplo, BR-1 representa un material preparado con la bentonita BR al que se agregó un 1 \% Ca mediante intercambio iónico. Los porcentajes de Ca agregados guardan relación con distintos porcentajes de CIC de BR (Tabla 1).

Para la modificación, se agregó a la arcilla sólida la solución de $\mathrm{Ca}^{2+}$ en sus respectivas concentraciones. Las suspensiones fueron agitadas durante $16 \mathrm{~h}$ a $25^{\circ} \mathrm{C}$. Posteriormente se realizaron lavados por centrifugación y se secaron a $70^{\circ} \mathrm{C}$. Los sólidos obtenidos se molieron y tamizaron pasante Malla 100. Con el objeto de determinar la cantidad de Ca retenido en la bentonita, se cuantificó dicho catión a partir de las fracciones sobrenadantes luego de los lavados. La concentración de $\mathrm{Ca}^{2+}$ sobrenadante fue determinada mediante un espectrofotómetro de absorción atómica (GBC 904 AA). La cantidad incorporada por el sólido se calculó como la diferencia entre el total de Ca inicialmente añadido para realizar el intercambio y el que se observó en solución. Además, se realizó la corrección de las concentraciones con un blanco de muestra. Los datos permiten evidenciar que la cantidad de $\mathrm{Ca}$ incorporado en la bentonita se mantiene prácticamente constante una vez alcanzado el equivalente al \% Ca necesario para compensar la CIC del material natural (2\% Ca).

Tabla 1: Retención de Ca en BR.

\begin{tabular}{|c|c|c|c|c|c|}
\hline & & \multirow[t]{2}{*}{ NOMENCLATURA } & \multicolumn{2}{|c|}{$\begin{array}{l}\text { Ca INCORPORADO } \\
(\% \mathrm{p} / \mathrm{p})\end{array}$} & \multirow[t]{2}{*}{$\% \mathrm{CIC}$} \\
\hline & & & TEÓRICO & EXPERIMENTAL & \\
\hline \multirow{4}{*}{$\begin{array}{l}\text { Ca AGREGADO } \\
(\% p / p)\end{array}$} & 1 & BR-1 & 1,0 & 0,9 & 50 \\
\hline & 2 & BR-2 & 2,0 & 1,8 & 100 \\
\hline & 3 & BR-3 & 2,0 & 2,0 & 150 \\
\hline & 4 & BR-4 & 1,9 & 1,9 & 250 \\
\hline
\end{tabular}

Dada la menor cantidad de Ca agregado con un resultado de incorporación semejante a las demás, la muestra elegida para posteriores estudios fue la BR-2, la cual fue renombrada como BR-Ca.

Con el objeto de determinar cómo influye la presencia de $\mathrm{Ca}^{2+}$ interlaminar en el comportamiento desecante de las muestras bentoníticas modificadas se estudió la capacidad de adsorción de agua de dichas muestras.

\subsection{Determinación de la capacidad desecante}

Los ensayos de adsorción se llevaron a cabo a $25^{\circ} \mathrm{C}$ en ambientes cerrados entre $11-98 \%$ HR. Los valores de $\mathrm{HR}$ deseados, se obtuvieron con soluciones salinas saturadas ( $\mathrm{LiCl} \mathrm{HR} 11 \%, \mathrm{MgCl}_{2} \mathrm{HR} 33 \%, \mathrm{Mg}\left(\mathrm{NO}_{3}\right)_{2} \mathrm{HR}$ $56 \%, \mathrm{NaCl} \mathrm{HR} \mathrm{75 \% ,} \mathrm{y} \mathrm{K}_{2} \mathrm{SO}_{4} \mathrm{HR} 98 \%$ ). Previo a los ensayos de adsorción las muestras fueron secadas a $70^{\circ} \mathrm{C}$ durante $24 \mathrm{~h}$.

El ensayo consistió en colocar una cantidad conocida de muestra en el desecador a una HR apropiada. La cantidad de agua adsorbida se determinó mediante pesadas periódicas hasta obtención de masa constante [17].

El cálculo de la cantidad relativa de agua adsorbida, $\mathrm{q}_{\mathrm{e}}\left(\mathrm{g} \mathrm{g}^{-1}\right)$, se determinó a partir de la ecuación (1).

$$
q_{e}\left(g \times g^{-1}\right)=\frac{\left(m_{e}-m_{s}\right)}{m_{s}}
$$

donde me representa el peso de la muestra húmeda $(\mathrm{g})$ en el equilibrio y ms representa el peso de la muestra seca (g). 


\section{RESULTADOSY DISCUSIÓN}

\subsubsection{Difracción de Rayos X}

Los resultados de los análisis por DRX para BR y BR-Ca se presentan en la Figura 1. Se observa que los espaciados d(001), medidos a 55\% HR, corresponden a 14,5 y 15,0 A respectivamente, y están de acuerdo con lo reportado para materiales similares [7, 18]. La bentonita BR contiene en la intercapa iones $\mathrm{Na}^{+}, \mathrm{Ca}^{2+} \mathrm{y} \mathrm{K}^{+}$, quienes presentan diferentes estados de hidratación, resultando un pico de difracción ancho y asimétrico. Además, los iones $\mathrm{Na}^{+}$y $\mathrm{K}^{+}$, de baja energía de hidratación, dan lugar a una menor separación de las láminas de arcilla [18]. Durante el intercambio dichos iones son reemplazados por el ión $\mathrm{Ca}^{2+}$ debido a la alta capacidad de intercambio de este último. El aumento en el espaciamiento basal de la estructura en BR-Ca puede ser un indicador de que más iones $\mathrm{Ca}^{2+}$, de alta energía de hidratación, alcanzan los sitios de adsorción interlaminar. Asimismo, el pico de la muestra homoionizada se observa más estrecho y con mayor intensidad y simetría. Lo expresado anteriormente confirmaría la incorporación de los iones $\mathrm{Ca}^{2+}$ en los sitios interlaminares.

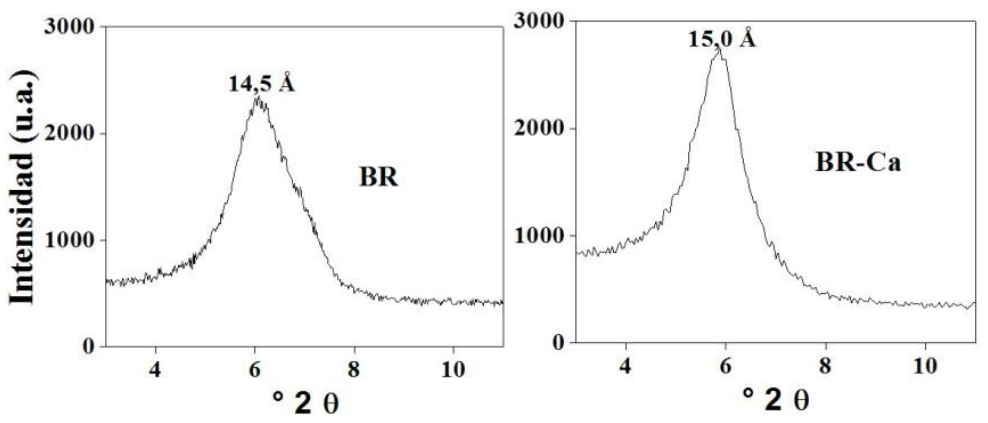

Figura 1: DRX de bentonita natural, BR, y modificada con Ca, BR-Ca.

La diferencia en el espaciamiento basal entre las dos muestras, $0,5 \AA$, seria debido a que la cantidad de $\mathrm{Ca}$, suficiente para saturar la CIC, es pequeña. Sin embargo, los valores son relevantes para aplicaciones como desecante de la bentonita modificada con $\mathrm{Ca}$.

\subsubsection{Espectroscopia Infrarroja}

En la Figura 2 se presentan los espectros FTIR de la bentonita BR y BR-Ca. Los picos a 3630 y $3438 \mathrm{~cm}^{-1}$ están bien diferenciados. La posición y forma de la banda de estiramiento del OH estructural $\left(3630 \mathrm{~cm}^{-1}\right) \mathrm{se}^{\mathrm{s}}$ ven influidas principalmente por la naturaleza de los átomos octaédricos a los que se coordinan los grupos hidroxilo. Además, debido a las diferencias en el radio, la carga y las energías de hidratación de los cationes intercambiables, el tipo de catión también influye en estas bandas, pero en menor medida [19]. Por todo esto se observa que ambas muestras presentan espectros con gran similitud. Sin embargo, en BR-Ca la banda a $3438 \mathrm{~cm}^{-1}$, debida a las vibraciones $\mathrm{H}-\mathrm{OH}$ del agua adsorbida sobre la estructura y / o agua interlaminar, aumenta su intensidad en relación a la banda del $\mathrm{OH}$ estructural $\left(3630 \mathrm{~cm}^{-1}\right)$. Esta es una manifestación del estado de hidratación de las muestras.

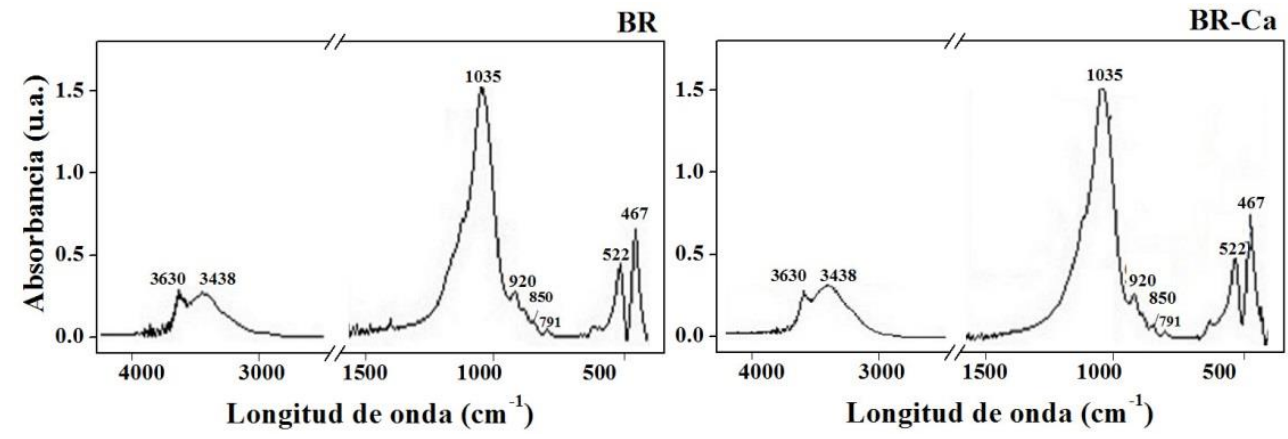

Figura 2: FTIR de bentonita natural, BR, y modificada con Ca, BR-Ca.

La banda intensa a $1035 \mathrm{~cm}^{-1}$ es debido al estiramiento del enlace Si-O-Si relacionado a los oxígenos 
basales.

Las bandas de flexión del $\mathrm{OH}$ en $920 \mathrm{~cm}^{-1}$ y $850 \mathrm{~cm}^{-1}$ son atribuidos a deformaciones de grupos Al$\mathrm{OH}$, y $\mathrm{Mg}-\mathrm{Al}-\mathrm{OH}$ respectivamente, lo cual confirma la presencia de esmectitas dioctaédricas, (sustitución parcial en el octaedro de $\mathrm{Al}$ por $\mathrm{Mg}$ ).

La señal en $791 \mathrm{~cm}^{-1}$ se asigna a la vibración Si-O característica de cuarzo [20], es decir sílice sin interacción con las láminas de montmorillonita. Asimismo, dicho cuarzo presenta bandas que se superponen con las bandas de Si-O a $1035 \mathrm{~cm}^{-1}$ de la capa tetraédrica presentes en la montmorillonita. Dicha banda se presenta con aproximadamente igual intensidad en ambas muestras debido a que durante el tratamiento no se realizó un proceso de purificación para eliminar el cuarzo. Dicho resultado se condice con $\% \mathrm{SiO}_{2}$ determinado por análisis químico (sección 3.1.3).

En la Tabla 2 se presenta la razón entre Absorbancias a $3438 \mathrm{~cm}^{-1}$ y $1035 \mathrm{~cm}^{-1}$ ( $\mathrm{A}_{3438} / \mathrm{A}_{1035}$ ), la cual está directamente relacionada con la hidrofilidad las muestras [19-20]. Se observa que dicha relación aumenta en BR-Ca indicando mayor grado de hidrofílico.

Tabla 2: Razón entre las Absorbancias relativas a $3438 \mathrm{~cm}^{-1}$ y $1035 \mathrm{~cm}^{-1}$.

\begin{tabular}{l|l|l}
\hline & BR & BR-Ca \\
\hline $\mathrm{A}_{3438} / \mathrm{A}_{1035}$ & 0,18 & 0,22 \\
\hline
\end{tabular}

Por último aparecen dos bandas a 518 y $465 \mathrm{~cm}^{-1}$ correspondiente a la vibración de los enlaces Si-OAl en la capa octaédrica y $\mathrm{Si}-\mathrm{O}$ en la capa tetraédrica respectivamente [20], las cuales son visibles en ambas muestras.

\subsubsection{Análisis químico}

En la Tabla 3 se informa la composición química y la pérdida por calcinación (PPC) de BR y BR-Ca. Los resultados se corresponden con valores reportados en la literatura para bentonitas con alto contenido de esmectita [5, 21-22] y con importante contenido de $\mathrm{Fe}_{2} \mathrm{O}_{3}$ típica de las llamadas ocres.

Tabla 3: Composición química y pérdida por calcinación (PPC) de BR y BR-Ca.

\begin{tabular}{l|l|l|l|l|l|l|l|l|l|l}
\hline & $\mathrm{SiO}_{2}$ & $\mathrm{Al}_{2} \mathrm{O}_{3}$ & $\mathrm{Fe}_{2} \mathrm{O}_{3}$ & $\mathrm{MnO}$ & $\mathrm{MgO}$ & $\mathrm{CaO}$ & $\mathrm{Na}_{2} \mathbf{O}$ & $\mathrm{K}_{2} \mathrm{O}$ & $\mathrm{TiO}_{2}$ & $\mathbf{P P C}$ \\
\hline BR & 57,71 & 18,12 & 4,79 & 0,05 & 2,95 & 1,66 & 2,63 & 0,34 & 0,23 & 10,76 \\
\hline BR-Ca & 57,18 & 18,67 & 4,84 & 0,04 & 2,95 & 2,78 & 0,89 & 0,27 & 0,23 & 13,05 \\
\hline
\end{tabular}

El análisis químico de BR arrojó presencia de calcio, sodio y potasio, correspondientes a cationes de intercapa de la esmectita y a feldespatos presentes [23]. Además, el $\mathrm{CaO}$ en BR-Ca fue de 2,78 \% en comparación con $1,66 \%$ en $\mathrm{BR}$, mientras que los contenidos de $\mathrm{Na}_{2} \mathrm{O}$ y $\mathrm{K}_{2} \mathrm{O}$ se redujeron luego de la modificación. Esto también indicó que más iones $\mathrm{Ca}^{2+}$ se incorporaron en la región interlaminar de la bentonita.

\subsubsection{Superficie específica BET}

En la Figura 3 se presentan las isotermas de adsorción- desorción de $\mathrm{N}_{2}$ a $77 \mathrm{~K}$ de las muestras en estudio. Se observa que BR-Ca, cuya isoterma es de tipo IV, sigue aproximadamente la misma trayectoria que la de su material de partida. 


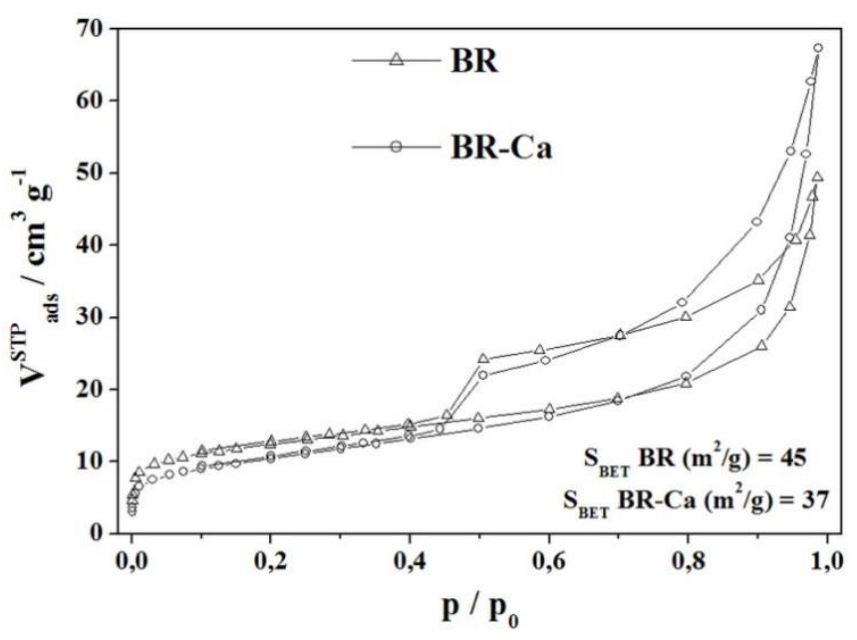

Figura 3: Isotermas de adsorción/desorción de $\mathrm{N}_{2}$ de BR y BR-Ca

En particular, en la rama de adsorción de BR-Ca se presenta menor adsorción de $\mathrm{N}_{2}$ con respecto a BR, hasta $\mathrm{p} / \mathrm{p}_{0}=0,8$. Luego la isoterma se desvía y comienza un ascenso más marcado. Al llegar a presiones cercanas a la de saturación, la pendiente de la isoterma sigue presentando un mayor aumento. Esto es atribuido a la presencia de mesoporos más grandes. Así también se observa que BR-Ca presenta menor $\mathrm{S}_{\mathrm{BET}}$ en relación a BR.

\subsection{Adsorción de vapor de agua en muestra natural y modificada}

En la Figura 4 se observan las isotermas de adsorción de agua, entre 11- $98 \%$ HR, de la bentonita natural y las modificadas (por agregado de Ca hasta un 2\%). Las mismas indicaron una estrecha relación entre el poder desecante y el contenido de $\mathrm{Ca}$ en la muestra arcillosa, confirmando la posibilidad de aplicación de estas muestras como materiales adsorbentes de humedad. Para un dado valor de HR, la adsorción aumenta con el porcentaje de $\mathrm{Ca}$ incorporado.

La mayor retención de las muestras modificadas, se atribuye a la mayor densidad de carga de los iones $\mathrm{Ca}^{2+}$, por lo cual es mayor la interacción con las moléculas de $\mathrm{H}_{2} \mathrm{O}$, en relación a los otros iones de intercapa $\mathrm{Na}^{+}$y $\mathrm{K}^{+}$[18], los cuales son desplazados durante el intercambio. Asimismo, como consecuencia del alto calor de hidratación del ión $\mathrm{Ca}\left(\mathrm{H}_{2} \mathrm{O}\right)_{6}{ }^{2+}{ }^{2}$ [2] en BR-Ca se induce un aumento en el espacio interlaminar de la arcilla, lo cual se condice con los valores d(001) determinados por DRX.

Así también, se observa en la Figura 4 que la adsorción aumenta rápidamente hasta el valor de $11 \%$ $\mathrm{HR}$, posteriormente hay una reducción en la velocidad de adsorción hasta aproximadamente el $56 \%$ HR. A partir de allí, se presenta un punto de inflexión, con un aumento de adsorción cuando aumenta el \% HR. Este comportamiento se mantiene a medida que aumenta el \% $\mathrm{Ca}$ agregado ya que las pendientes en cada trayecto de las isotermas son aproximadamente iguales. A medida que la HR aumenta por encima de $75 \%$ la adsorción aumenta nuevamente. En esta región comenzaría a formarse gradualmente la segunda capa de agua, dada la menor pendiente de las curvas [25-26]. En dichas condiciones de HR otras moléculas de agua se condensarían en la superficie de los tactoides, aumentando la cantidad de agua retenida. En este punto la curva de adsorción se vuelve nuevamente cóncava hacia arriba. 


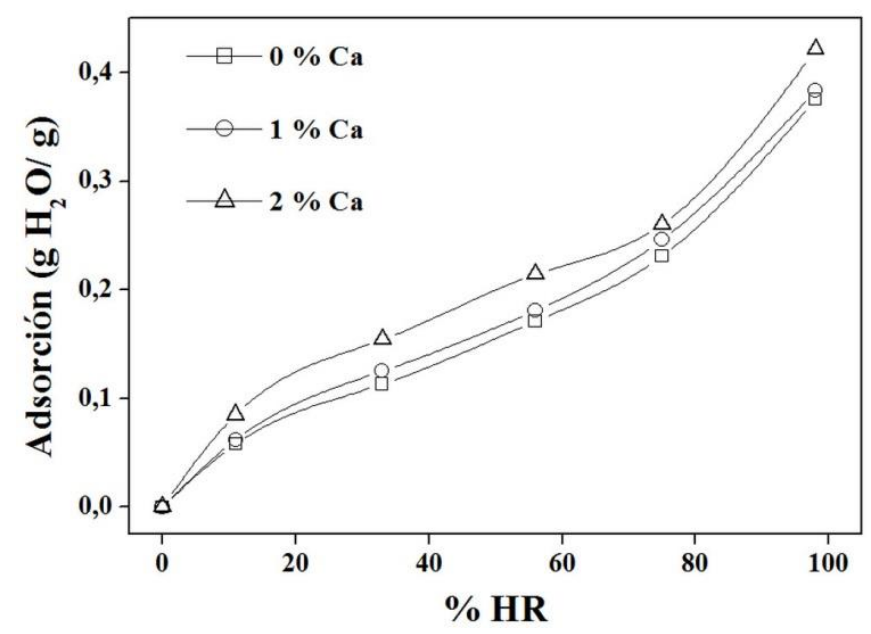

Figura 4: Isotermas de adsorción de vapor de agua de BR y para los distintos \% Ca agregado.

El buen comportamiento de adsorción por parte de las muestras en general, estaría relacionado con las características estructurales, tales como el contenido de sitios hidrofílicos, determinados por FTIR del mineral montmorillonita. En consecuencia, las mismas, tendrían menor cristalinidad en su estructura, lo cual las haría más reactivas. Asimismo, del análisis de $\mathrm{pH}$ alcalino de la suspensión arcillosa, y de acuerdo a la bibliografía [25-26], las cargas negativas se neutralizarían con la adsorción de cationes hidratados y moléculas de agua.

El aumento de adsorción luego de la modificación, aumenta con el aumento del \% HR. Esto podría sugerir que a altas HR la muestra natural y modificada presentan un buen comportamiento desecante.

\section{CONCLUSIONES}

La bentonita estudiada es un buen adsorbente de agua. A $98 \% \mathrm{HR}$ y $25{ }^{\circ} \mathrm{C}$ el valor de retención de BR es $0,375 \mathrm{~g} \mathrm{H}_{2} \mathrm{O} / \mathrm{g}$ bentonita.

Los análisis químicos indicaron que en $\mathrm{BR}-\mathrm{Ca}$, el $\mathrm{Ca}^{2+}$ desplazó al $\mathrm{Na}^{+} \mathrm{y} \mathrm{K}^{+}$presentes originalmente.

Un incremento de adsorción es observado cuando se aumenta el contenido de Ca en la región interlaminar de la esmectita. A $98 \% \mathrm{HR}$ y $25^{\circ} \mathrm{C}$, BR-Ca alcanzó a retener 0,396 $\mathrm{g} \mathrm{H}_{2} \mathrm{O} / \mathrm{g}$ bentonita.

La modificación realizada, mediante un simple tratamiento de intercambio iónico, contribuye a una mejora de la aplicación como desecante de la arcilla de procedencia territorial. Esto podría jugar un rol importante en la posibilidad de aplicaciones a nivel industrial, que requieren desecantes en cantidad y cuyo precio se determina más por el proceso de preparación que por el precio de las materias primas.

\section{AGRADECIMIENTOS}

Los autores agradecen a las instituciones que hacen posible el desarrollo de esta investigación a través del proyecto $N^{\circ} 2048$, Consejo de Investigación de la Universidad Nacional de Salta (CIUNSa) y CONICET.

\section{BIBLIOGRAFÍA}

[1] MARI E. A., Los Materiales Cerámicos, 1 ed., Argentina, Alsina, 1998.

[2] RODRÍGUEZ, L. D., SAN MILLÁN, R. T., “Arcillas cerámicas: una revisión de sus distintos tipos, significados y aplicaciones”, Boletín de la Sociedad española de Cerámica y Vidrio, v. 41, n. 5, pp. 459-470, Oct. 2002.

[3] NEAMAN, A., PELLETIER, M., VILLIERAS, F., "The effects of exchanged cation, compression, heating and hydration on textural properties of bulk bentonite and its corresponding purified montmorillonite", Applied Clay Science, v. 22, n. 4, pp. 153-168, Feb. 2003.

[4] MONTES-H, G., GERAUD, Y., "Sorption kinetic of water vapour of MX80 bentonite submitted to different physical-chemical and mechanical conditions", Colloids and Surfaces A: Physicochemical and Engineering Aspects, v. 235, n. 1, pp. 17-23, March 2004. 
[5] BULUT, G., CHIMEDDORJ, M., ESENLI, F., et al., "Production of desiccants from Turkish bentonites", Applied Clay Science, v. 46, n. 2, pp. 141-147, Oct.2009.

[6] MEKHAMER, W. K., "Energy storage through adsorption and desorption of water vapour in raw Saudi bentonite", Arabian Journal of Chemistry, v. 9, pp. S264-S268, Sep. 2016.

[7] MACEWAN, D. M., WILSON, M. J., "Interlayer and intercalation complexes of clay minerals", In: Brindley, G. W., Brown, G.(eds), Crystal Structures of Clay Minerals and their X-Ray Identification, chapter 3, London, Mineralogical Society, 1980.

[8] ODOM, I. E, "Smectite clay minerals: properties and uses", Philosophical Transactions of the Royal Society of London. Series A, Mathematical and Physical Sciences, v. 311, n. 1517, pp. 391-409, Jun.1984.

[9] GRIM, R. E., GUVEN, N., Bentonites: geology, mineralogy, properties and uses, The Netherlands, Elsevier, 2011.

[10] ÇINAR, M., ERSEVER, G., ŞAHBAZ, O., ÇELIK, M. S., "Sepiolite/calcium interactions in desiccant clay production", Applied Clay Science, v. 53, n. 3, pp. 386-394, Sep. 2011.

[11] THORUWA, T. F. N., JOHNSTONE, C. M., GRANT, A. D., et al., "Novel, low cost $\mathrm{CaCl}_{2}$ based desiccants for solar crop drying applications", Renewable energy, v. 19, n. 4, pp. 513-520,April 2000

[12] MACAS, A, Absorción y desorción de vapor de agua mediante la utilización de las zeolitas naturales de la provincia de guayas. Tesis de grado, ESCUELA SUPERIOR POLITÉCNICA DEL LITORAL, Guayaquil- Ecuador, 2012.

[13] YE, W. M., CUI, Y. J., QIAN, L. X., et al., "An experimental study of the water transfer through confined compacted GMZ bentonite”, Engineering Geology, v. 108, n. 3, pp. 169-176,Oct. 2009.

[14] ARISTOV, Y. I., RESTUCCIA, G., CACCIOLA, G., et al., "A family of new working materials for solid sorption air conditioning systems", Applied Thermal Engineering, v. 22, n. 2, pp. 191-204, Feb.2002.

[15] TRETIAK, C. S., ABDALLAH, N. B., "Sorption and desorption characteristics of a packed bed of clay$\mathrm{CaCl}_{2}$ desiccant particles", Solar Energy, v. 83, n. 10, pp. 1861-1870, Oct. 2009.

[16] ROUQUEROL, J., ROUQUEROL, F., LLEWELLYN, P., et al., Adsorption by powders and porous solids: Principles, Methodology and Applications, 2 ed., France, Academic press Elsevier, 2013.

[17] Norma española UNE-EN ISO 12571, "Prestaciones higrotérmicas de los productos y materiales para edificación. Determinación de las propiedades de sorción higroscópica”, 2015.

[18] CANCELA, G. D., HUERTAS, F. J., TABOADA, E. R., et al., "Adsorption of water vapor by homoionic montmorillonites. Heats of adsorption and desorption", Journal of colloid and interface science, v.185, n. 2, pp. 343-354, Jan. 1997.

[19] MADEJOVÁ, J., "FTIR techniques in clay mineral studies", Vibrational spectroscopy, v. 31, n. 1, pp. 110,Jan. 2003.

[20] DE BRAVO, Y. O., GÓMEZ, C., DE RAMÍREZ, I., "Análisis por espectroscopia infrarroja de algunas arcillas colombianas", Revista Colombiana de Química, v. 10, n. 1, pp. 45-52, 1980.

[21] AOCHI, Y. O., FARMER, W. J., "Effects of surface charge and particle morphology on the sorption/desorption behavior of water on clay minerals", Colloids and Surfaces A: Physicochemical and Engineering Aspects, v. 374, n. 1, pp. 22-32, Nov. 2011.

[22] VOLZONE C., ORTIGA, J., "Influence of the exchangeable cations of montmorillonite on gas adsorptions", Process Safety and Environmental Protection, v. 82 (B2), pp. 170-174, Mar. 2004.

[23] MERMUT, A. R., CANO, A. F., "Baseline studies of the clay minerals society source clays: chemical analyses of major elements", Clays and Clay Minerals, v.49, n. 5, pp. 381-386, Oct. 2001.

[24] CASTRILlO, N., MERCADO, A., VOLZONE, C., "Estimación de la energía de hidratación de desecantes bentoníticos", In: Anales de las XI Jornadas de Ciencia y Tecnología de Facultades de Ingeniería del NOA, pp. 706-710, San Salvador de Jujuy, Jun. 2016.

[25] FERRAGE, E., LANSON, B., SAKHAROV, B. A., et al., "Investigation of smectite hydration properties by modeling experimental X-ray diffraction patterns: Part I. Montmorillonite hydration properties", American Mineralogist, v. 90(8-9), pp. 1358-1374, Aug. 2005.

[26] VIEILLARD, P., BLANC, P., FIALIPS, C. I., et al., "Hydration thermodynamics of the SWy-1 montmorillonite saturated with alkali and alkaline-earth cations: a predictive model", Geochimica et Cosmochimica Acta, v.75, n. 19, pp. 5664-5685, Oct. 2011. 